\title{
Unternehmen Zukunft
}

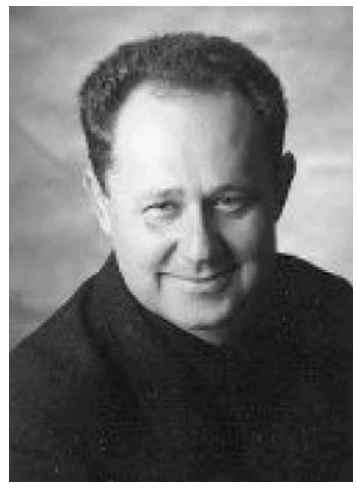

Prof. Dr. Manfred Wildner
Bibliografie

DOI http://dx.doi.org/

10.1055/s-0030-1267943

Gesundheitswesen 2010;

72: 761-762

(c) Georg Thieme Verlag KG Stuttgart · New York

ISSN 0941-3790

Korrespondenzadresse

Prof. Dr. M. Wildner

Bayerisches Landesamt

für Gesundheit und

Lebensmittelsicherheit

Veterinärstraße 2

85762 Oberschleißheim

Manfred.Wildner@Igl.bayern.de
„Scotty, beam uns hoch - es gibt kein intelligentes Leben auf diesem Planeten!“. Dieser kurze Dialog einer kleinen Expedition auf einem unbekannten Planeten mit dem Mutterschiff bezieht sich auf die imaginären Reisen des Raumschiffs Enterprise durch die Tiefen des Weltalls, weit in der Zukunft. Der Satz wird ironisch gelegentlich auch auf unseren Planeten angewandt. Intelligentes Leben auf der Erde - sieht es wirklich so schlecht aus? Manche der beim Raumschiff Enterprise zu findenden Zukunftsvisionen, wie die mobile Telephonie, sind heute eingeholt bzw. überholt.

Die Zukunft als Singular? Schon an dieser Stelle ist einer naiven Zukunftserwartung zu widersprechen. Zukunft ereignet sich nicht unausweichlich wie die Ankunft an der jeweiligen nächsten Haltestelle einer Bahnfahrt, sie wartet nicht „fertig“ auf uns. Auch prophetische Äußerungen sind bei genauerem Hinsehen meist konditional in einen „wenn - dann“ Kontext gestellt. Es liegen „viele Zukünfte“ vor uns: Bessere Zukünfte, schlechtere Zukünfte. Bibliotheken und Videotheken sind voll von technisch-wissenschaftlichen und gesellschaftlichen Zukunftsfantasien. Sie reichen von Francis Bacons hellsichtiger „Utopia“ (1516) einer wissenschaftsbasierten humanen Gesellschaft über die Vorwegnahme einer fortschreitenden Evolution und verlängerten Lebensspanne (Franz Werfels „Stern der Ungeborenen“, 1946) zur düster-totalitären Vision „1984“ George Orwells (1949) und der bedrückenden Option einer schmerzfreien „Schönen neuen Welt" (1932) Aldous Huxleys. Sie erzählen von Sternfahrten (Raumschiff Enterprise, seit 1964), freundlichen extraterrestrischen Wesen (E.T., 1982), und künstlichem Leben in bedrohlichen (Matrix, 1999) bzw. verführerisch-schönen Parallelwelten bzw. Parallelkörpern (Avatar, 2009).

Was sind wissenschaftlich-technische $\mathrm{Zu}$ kunftsentwürfe für die Medizin? Neue Entwicklungen sind sowohl als Innovationen aus der Forschung heraus als auch von den Akteuren und Institutionen in der Praxis als neue Anfrage bzw. neuer Bedarf zu erwarten. Innovationen können die Akteure im Gesundheitswesen - Patienten, Leistungserbringer, Finanziers - unterschiedlich stark betreffen. Und auch den sogenannten „zweiten Gesundheitsmarkt“: Darunter werden die privat finanzierten Waren und Dienstleistungen verstanden, von frei verkäuflichen Arzneimitteln über individuelle Gesundheitsleistungen bis hin zu Sport, Fitness und Wellness. Nach Angaben des Bundesministeriums für Gesundheit wurden im Jahr 2005 knapp 55 Mrd. EUR dort ausgegeben [1].
Technologische Forschritte prägen die Medizin. Beispiele dazu sind minimal invasive Operationstechniken mit zunehmenden Indikationsausweitungen (z.B. Bandscheibenentfernungen, Gallenblasenentfernungen, Blinddarmentfernungen). Die Miniaturisierung hat eine weitere Ausprägung in der sogenannten Nanomedizin. Nanopartikel werden als Hüllsubstanzen für Arzneimittel eingesetzt, zur spezifischen Zielfindung therapeutischer Wirkstoffe im Gewebe und für die Diagnostik und Therapie im Zusammenhang mit externen Apparaturen zur verbesserten Bildgebung oder auch gezielteren Krebsbekämpfung. In der Diagnostik kommt der schnellen Testung zunehmend Bedeutung zu. Beispiele dafür sind HIV-Schnelltestungen, welche als bed-side-Tests oder point-of-care-Tests durchgeführt werden können. Gleichzeitig führt die Miniaturisierung dazu, dass anhand einer einzigen Blutprobe eine Vielzahl von diagnostischen Tests zeitgleich durchgeführt werden können. Dies eröffnet Möglichkeiten hinsichtlich der gesundheitlichen Prognose bei bestehender Krankheit, dem Screening und auch der Prädiktion. Unter prädiktiver Medizin versteht man den Versuch, individuelle Krankheitsrisiken vorherzusagen, im Idealfall ohne dass sich bereits Vorstufen von Erkrankungen finden. Derartige Herangehensweisen sind auch kritisch zu betrachten, da sie von einer Verunsicherung der betroffenen Personen begleitet sein können, ohne dass sichere Aussagen über das Auftreten oder Nicht-Auftreten einer Krankheit bislang möglich wären (s. §18 GenDG).

Die Automatisierung führt auch zu verbesserten bildgebenden Verfahren, wie z. B. Ganzkörper-Tomografien zu Screeningzwecken. Es ist zu erwarten, dass die Ausweitung der Diagnostik einerseits zu einer wünschenswerten Früherkennung von behandlungsbedürftigen Erkrankungen führt, andererseits aber auch zur Erkennung von nicht behandlungsbedürftigen, medizinisch wenig relevanten Befunden, welche dann einer unnötigen Behandlung zugeführt werden, oder aber zu falsch positiven Befunden mit hohen Folgekosten. Innovationen können grundsätzlich auch zu Kosteneinsparungen führen. Ob derartige potenziell kostensenkende Innovationen, die auch als „disruptive technologies“ bezeichnet werden, tatsächlich eine Chance in einem auf Gewinn ausgerichteten Gesundheitsmarkt haben, hängt stark von den Rahmenbedingungen ab [2]. Oft kommen Sie als „add-on“-Technologien sukzessiv und kostenrelevant mit jeweils marginalem zusätzlichem Informationsgewinn zum Einsatz (Beispiel: normales Röntgenbild, Ultraschall, CT, NMR, PET-Scan). 
Große Hoffungen werden auch auf eine individualisierte Prävention und Therapie gesetzt. Der irreführende Begriff der „Personalisierung“ sollte hier nicht verwendet werden, der integrativen, ganzheitlichen und gesprächsorientierten Ansätzen in der Medizin vorbehalten ist. Die zugehörigen Verfahren werden als Omics-Technologien bezeichnet und haben begriffliche Ausprägungen als Proteomics, Genomics, Metabonomics, Metabolomics, Transkriptomics, Metallomics, Glycomics und anderes mehr.

Erhebliche Forschungsanstrengungen werden zur Züchtung von biologischem Gewebe oder auch ganzer Organe unternommen. Teilweise sind derartige Verfahren bereits im Einsatz, wie z.B. die Autologe Chondrozyten Transplantation. Eine Kombination von Automatisierung und regenerativer Medizin sind die Integration technischer Module in den menschlichen Körper. Erfolgreiche Produkte sind die bekannten Hüft- und Knieprothesen. Diese Technologie wird zunehmend auch auf andere Gelenke ausgeweitet und auch erfolgreich bei anderen Organen eingesetzt (künstliche Herzklappe, Forschungen zu Neuroprothesen, künstlicher Blutersatz).

Die genannten Veränderungen werden voraussichtlich die Leistungserbringung im Rahmen von integrierten Versorgungsmodellen befördern. Gründe dafür sind zum Einen hohe Bereitstellungskosten für hoch spezialisierte Technologien, zum Zweiten zunehmende Abwanderungen von bisher stationär durchgeführten Eingriffen in den ambulanten Bereich bei gleichzeitigem Bedarf an stationären back-up Kapazitäten zur Komplikationsbehandlung und zum Dritten zur Qualitätsverbesserung bei gleichzeitiger Effizienzsteigerung der Patientenbehandlung durch integrierte Konzepte. Im Rahmen einer zunehmenden Medialisierung von Risiken medizinischer Behandlung, z. B. Krankenhausinfektionen mit multiresistenten Erregern oder Arzneimittelinteraktionen, wird erwünschtes Bewusstsein der Notwendigkeit eines aktiven Risikomanagements der ambulanten und stationären Therapie zunehmen. Solches proaktives Risikomanagement wird auch von der europäischen Ebene befördert (z.B. Luxemburger Erklärung zur Patientensicherheit [3]).

Eine starke Kraft ist in diesem Zusammenhang die Evidenz-basierte Medizin. Seitens der Gesetzlichen Krankenversicherung (GKV) wurde 2006 das Innovationsmanagement der GKV ins Leben gerufen, welches mit der medizinischen Expertise des Medizinischen Dienstes des Spitzenverbandes Bund der Krankenkassen (MDS) vernetzt ist. In der Ärzteschaft wird in diesem Zusammenhang das Problem der Fremdbestimmung durch andere Berufsgruppen thematisiert. Eine wichtige Rolle könnte sich dabei für den Öffentlichen Gesundheitsdienst (ÖGD) als überparteilichen Sachwalter ergeben.

Aufgrund eines allgemein besseren Bildungsstandards, dem leichten Zugriff auf medizinische Fachinformationen auch für Laien bzw. Patienten und deren Angehörige sowie eine zunehmende gesellschaftliche Demokratisierung sind Veränderungen im ArztPatienten-Verhältnis angestoßen. „Mündige Patienten“ treffen diagnostische oder therapeutische Entscheidungen im Gespräch und gemeinsam mit dem Arzt (shared decision making bzw. partizipatorische Entscheidungsfindung). Teilweise wird auch der „fordernde Patient" und der „falsch informierte Patient" thematisiert. Patientenfürsprecher z.B. in Krankenhäuser sind eine weitere Ausprägung der Demokratisierungstendenzen.

Zusammenfassend ist für die technologische Forschung eine Kombination von Miniaturisierung, (Bio)technologischer Weiterentwicklung/Automatisierung und Individualisierung kenn- zeichnend, für die medizinische Versorgung eine Hinwendung zu präventiver, prädiktiver, personalisierter und partizipatorischer (System-)Medizin, welche teilweise von privaten Haushalten finanziert wird $[4,5]$. Wir sind, als wissensbasierte Gesellschaft, gut beraten, uns strukturiert mit Gegenwart und Zukunft der Medizin zu befassen - und mit ihren Grenzen, nicht nur den Grenzen des Machbaren, sondern besonders auch den Grenzen des Wünschenswerten und auch des Erlaubten. Unter systematischen Herangehensweisen finden sich Health Technology Assessment (HTA) und Health Impact Assessment (HIA), Prognosen und Modellrechnungen, Memoranden und Strategiepapiere und auch Institutionen wie der gemeinsame Bundesausschuss, das Institut für Qualität und Wirtschaftlichkeit im Gesundheitswesen und der Ethikrat.

Beispiel für die kritische Auseinandersetzung mit Handlungsoptionen in der Suche nach einer guten, womöglich besseren $\mathrm{Zu}$ kunft, finden sich in den Beiträgen dieses Heftes: Es geht um Hilfekonferenzen für psychisch Kranke und ambulante psychiatrische Behandlungen, die Früherkennung der Schizophrenie, um Palliativbehandlung und Qualitätsberichte in der Pflege, um Selektionsanreize des Risikostrukturausgleichs und sozioökonomische Wohnfeldindikatoren, um Gesundheitsuntersuchungen und - mit einem Blick über die Grenzen - um Schmerzmitteleinnahme bei Oberstufenschülern bei unserem europäischen Nachbarn Polen.

Was ist das Ziel all dieser Bemühungen? Vielleicht sind die Fahrten des Raumschiffes Enterprise Symbol für das Unternehmen Zukunft ganzer Gesellschaften. Mit Recht wurde unser Planet Erde auch als „Spaceship Earth“ (Raumschiff Erde) bezeichnet [6]. Kenneth Boulding wollte mit dieser Metapher nachdrücklich darauf hinweisen, dass wir uns bei genauerem Hinsehen nicht in einem unbegrenzten Raum bewegen, sondern in einem sphärisch geschlossenen, begrenzten und verwundbaren Lebensraum: Dies gilt für die „Ökosphäre“ genauso wie für eine „Ökonosphäre“ und eine „Soziosphäre“. Es geht bei unserem irdischen „Unternehmen Zukunft“ daher nicht nur um die Suche nach intelligenten technischen Problemlösungen, Möglichkeiten und Horizonten. Es geht darüber hinaus und vor allem um die Gestaltung einer Welt, in der wir und andere Menschen gerne leben, arbeiten und lieben wollen - in Gesundheit und auch mit Krankheit und Behinderung [7]. Solange wir gemeinsam unterwegs sind.

\section{Literatur}

1 Bundesministerium für Gesundheit. Zukunftskongress Gesundheitswirtschaft: Zahlen und Fakten. Bonn, BMG; 2007

2 Christensen CM, Bohmer R, Kenagy J. "Will Disruptive Innovations Cure Health Care?" Harvard Business Review. 2000, URL: http://hbr.org/web/ extras/insight-center/health-care/will-disruptive-innovations-curehealth-care Zugriff am 01.10.2010

3 European Commission. Making it happen! Luxembourg Declaration on patient Safety. 2005, URL: http://ec.europa.eu/health/ph_overview/ Documents/ev_20050405_rd01_en.pdf Zugriff am 01.10.2010

4 Hood L, Heath JR, Phelps ME et al. Systems Biology and New Technologies Enable Predictive and Preventative Medicine. Science 2004; 306: $640-643$

5 Weston $A D$, Hood L. Systems biology, proteomics, and the future of health care: toward predictive, preventative, and personalized medicine. J Proteome Res 2004; 3: 179-196

6 Boulding KE. The economics of the spaceship earth. 1966, URL: http:// dieoff.org/page160.htm Zugriff am 01.10.2010

7 Hengsbach F. „Mehr Markt“ macht nicht gesund - Gesellschaftliche Risiken und solidarische Sicherung entsprechen einander. Gesundheitswesen 2008; 70: 339-349 mit Kommentaren 